\title{
Refined upper bounds on the coarsening rate of discrete, ill-posed diffusion equations
}

\author{
Selim Esedoḡlu ${ }^{1}$ and Dejan Slepčev ${ }^{2}$ \\ ${ }^{1}$ Department of Mathematics, University of Michigan, 530 Church St Ann Arbor, \\ MI 48109, USA \\ 2 Department of Mathematical Sciences, Carnegie-Mellon University, Pittsburgh, PA 15213, USA \\ E-mail: esedoglu@umich.edu and slepcev@math.cmu.edu
}

Received 18 March 2008, in final form 27 August 2008

Published 13 November 2008

Online at stacks.iop.org/Non/21/2759

Recommended by A L Bertozzi

\begin{abstract}
We study coarsening phenomena observed in discrete, ill-posed diffusion equations that arise in a variety of applications, including computer vision, population dynamics and granular flow. Our results provide rigorous upper bounds on the coarsening rate in any dimension. Heuristic arguments and the numerical experiments we perform indicate that the bounds are in agreement with the actual rate of coarsening.
\end{abstract}

Mathematics Subject Classification: 35K55

(Some figures in this article are in colour only in the electronic version)

\section{Introduction}

We study coarsening phenomena observed in discrete, ill-posed diffusion equations. These equations appear in a variety of settings: in models for granular flow where they describe the formation of shear bands in a granular material undergoing anti-plane shear [23], in image processing where they constitute one of the most well-known models-called the PeronaMalik method-for denoising images while preserving their edges [20,21] and in population dynamics where they describe the chemotactic motion of certain types of bacteria [11, 14, 18]. An upper bound for the coarsening rate for these equations in one and two dimensions was obtained in [6], and appeared to be optimal for the specific nonlinearity $(R$ in (1)) focused on there. The results of this paper constitute a significant improvement in two respects: the bounds are proven in any dimension and, more importantly, the new bounds depend on the nature of the nonlinearity; in particular, we believe that the present bounds are optimal (in terms of the leading order rate) for a large class of nonlinearities. 
The specific equation we focus on is from population dynamics $[11,14,18]$, and can be viewed as a natural, spatially discrete version of the partial differential equation

$$
v_{t}=\Delta R(v)=R^{\prime}(v) \Delta v+R^{\prime \prime}(v)|\nabla v|^{2} .
$$

However, in the applications we consider, the function $R(\xi): \mathbb{R} \rightarrow \mathbb{R}$ satisfies the property

$$
R^{\prime}(\xi)<0 \text { for all large enough }|\xi|,
$$

which makes equation (1) backwards parabolic whenever the magnitude $|v|$ of the solution is large enough. Consequently, there is no complete well-posedness theory for (1). Indeed, numerical experiments with discrete versions of (1) indicate very sensitive dependence on initial conditions [15].

In one space dimension, equation (1) is very closely related to the following ill-posed diffusion equation that plays a prominent role in image processing [20,21], and appears also in models of granular flow [23]:

$$
u_{t}=\left(R\left(u^{\prime}\right)\right)^{\prime} .
$$

The function $R$ in these applications also satisfies property (2). The connection with (1) is straightforward: setting $v=u^{\prime}$, we see that $v$ satisfies (1) if $u$ satisfies (3). In image processing, the following two (or higher) dimensional version of (1) was proposed by Perona and Malik [20,21] as an image segmentation and denoising technique:

$$
u_{t}=\left(R^{\prime}\left(u_{x}\right)\right)_{x}+\left(R^{\prime}\left(u_{y}\right)\right)_{y} .
$$

The goal of segmentation is to recognize the regions occupied by distinct objects in the scene depicted by a given image. Perona and Malik proposed (4) as a means of simplifying this task by replacing the given image, which is taken to be the initial condition for (4), by a crude, 'cartoon-like' approximation in which small scale details have been eliminated, and small regions have been merged to form few larger ones. The same instability issues seen in (1) also plague (4). In particular, there is no complete well-posedness theory for (4), either, despite a number of important steps in that direction [4, 8-10, 12, 24]. Some studies of (4) have therefore focused on its discrete in space version [6,7], and others on its regularizations $[1-3,5,16]$.

Despite their unstable behaviour, discrete versions of (1) and (4) find widespread use in the aforementioned applications - the sensitive dependence on initial data appear to be of secondary importance in these applications. In this paper we concentrate on the discrete-inspace, continuum in time version of (1), which after all are the true form of the models as they appear in applications. This in particular allows us to avoid the well-posedness issues. The precise form of the discretizations we work with are given in section 3.1.

Let us also note that one should expect the general approach we take to be applicable if one considers a regularized version of (1), as long as gradient-descent structure is maintained in the regularized model. Adding a regularization to the problem would introduce a new length scale, controlled by a regularization parameter. Such a length scale is introduced by the size of a grid cell in the discretized version. We set this length scale to 1 .

In numerical experiments with discretized versions of (1), initial data quickly develop into spikes that contain most of the mass of the solution. These spikes are supported on a single grid cell, do not move, but nevertheless interact with each other through a background where the solution has small magnitude. To be more specific, during this interaction, small spikes get absorbed by nearby larger ones, leading to an overall configuration of larger but fewer spikes as the evolution progresses; see figure 1 . This is the coarsening process that we study in this paper. In experiments with (3), the role of the spikes is played instead by jumps separated by 'terraces' on which the solution is approximately constant. The coarsening process merges the terraces to form fewer of them, which are then separated by larger jumps; see figure 2 . In 

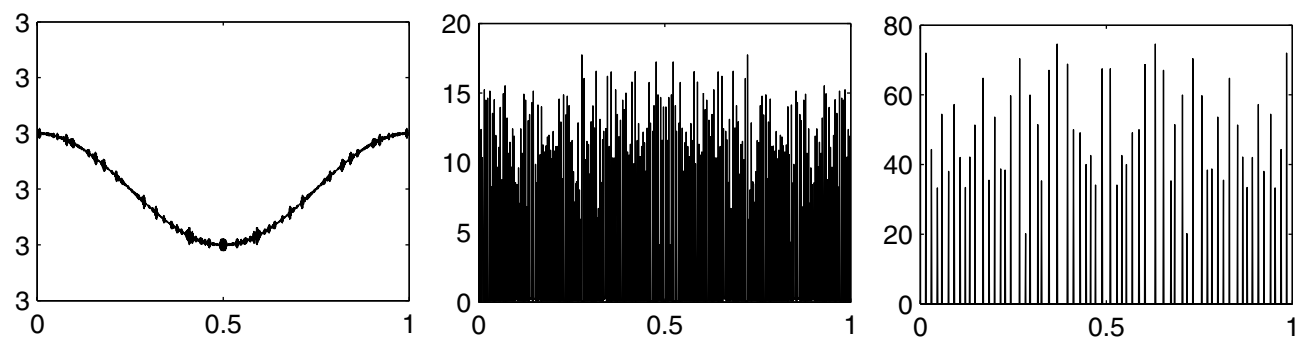

Figure 1. Spike formation and coarsening by the discrete version of equation (1), with $R(\xi)=$ $\xi /\left(1+\xi^{2}\right)$, in one space dimension. The solution is shown at times $t=6.67 \times 10^{-5}, t=3.33 \times 10^{-4}$ and $t=1.33 \times 10^{-2}$
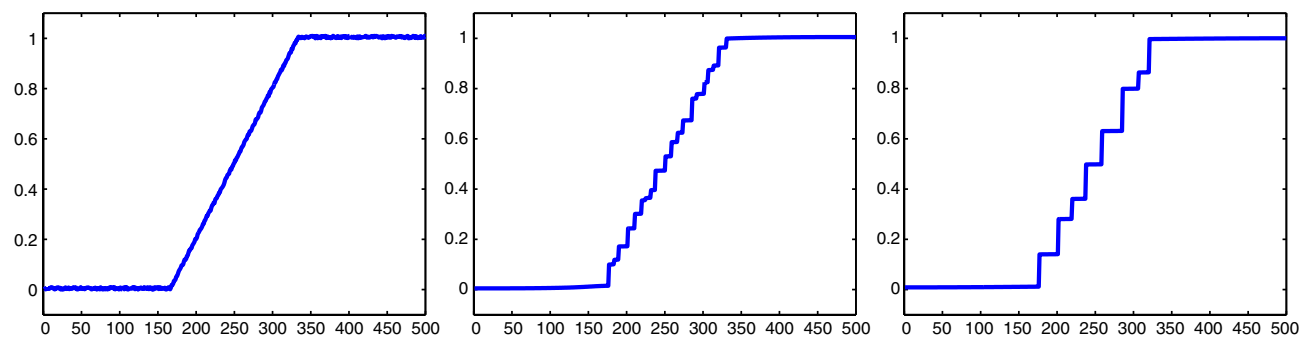

Figure 2. Formation and coarsening of terraces during the evolution by the Perona-Malik model. After a long time, there is often a single jump left separating two large terraces. This process is called 'edge enhancement' in image processing applications, in which a gradual transition is turned into a sharp discontinuity. In applications, the precise location of the final jump within the initial transition region is not as important as the rate at which the process takes place.

image processing, this behaviour is known as 'edge enhancement', because it turns a moderate transition in the image into a sharp discontinuity.

In many applications, for example in image processing via the Perona-Malik model, the rate at which this coarsening process takes place is of primary interest. Indeed, in this application, the coarsening rate reflects how fast a given image gets 'simplified' by the model. Understanding this rate is crucial in automatic selection of parameters, such as how long the diffusion process should be carried out on a given image. On the other hand, the precise arrangement of terraces and edges separating them, which seems to depend very sensitively on initial data as is to be expected from an ill-posed diffusion equation, is often not of critical significance: slight variations in edge locations can be tolerated by most applications that rely on processed images.

The main result of the paper is given in theorem 1 . It roughly says that if $R=f^{\prime}$ where $f(z) \sim z^{\alpha}$ for some $\alpha \in[0,1)$ for $z$ large, then the typical spike height scales as

$$
h \sim \begin{cases}t^{\frac{1}{3-\alpha}} & \text { in dimension } 1, \\ t^{\frac{1}{2-\alpha}} & \text { in dimension } d \geqslant 2 .\end{cases}
$$

The typical distance between nearby spikes scales as $h^{1 / d}$. Note that the coarsening is faster if $\alpha$ is closer to 1 . 
Outline. In section 2 we recall the previous work on the problem and the Kohn-Otto technique. In section 3 we introduce the precise setup. The main results on the rate of coarsening are proved in section 4 . In section 5 we present numerical experiments that support the conjecture that the upper bounds obtained are optimal for a large class of nonlinearities. Conclusions are briefly reviewed in section 6.

\section{Previous work}

Our approach is based on the technique of Kohn and Otto [13] for obtaining upper bounds on the coarsening in energy-driven systems. A bound on the rate of coarsening follows from estimates on the geometry of the energy landscape. In particular, in addition to the energy, $E$, one considers a quantity, $L$, which carries information on the length scales present in a configuration. The geometric information is contained in two inequalities relating $L$ and $E$ :

(1) Dissipation inequality

$$
\left(L^{\prime}\right)^{2} \leqslant C\left(-E^{\prime}\right)
$$

where $C$ is a universal constant.

(2) Interpolation inequality. When $E \ll 1$

$$
E L^{\beta} \geqslant C
$$

where $\beta>0$ and $C$ is again a universal constant.

If the equation is a gradient flow (as is the case for the systems we consider) then the natural choice for $L$ is the distance to a fixed configuration. The distance on the configuration space is induced by the inner product with respect to which the equation is a gradient flow. This makes the energy dissipation inequality immediate. Once the interpolation inequality is established, the version of the Kohn-Otto technique presented in [17] leads to the desired lower bound on (the time average of) the energy.

The techique of Kohn and Otto has been used in [6] to obtain upper bounds on coarsening rate for (1) in one and two spatial dimensions. In particular, the coarsening rate for the discrete version of (1) obtained in [6], which corresponds to $\alpha=0$ in (5), appears to be close to optimal in the case of the specific nonlinearity

$$
R(\xi)=\frac{\xi}{1+\xi^{2}}
$$

This nonlinearity is one of the common choices in applications (certainly in image processing, where it is the original choice of Perona and Malik in [20,21], but also in granular flow [23]). However other choices of $R$ that also lead to backwards parabolic behaviour are routinely used in applications. For most of other nonlinearities $R$, the bounds (and arguments) of [6] are not optimal, whereas the improved bounds obtained in this paper appear to be. Moreover, the results of this paper are more general, presented in any space dimension. The practical implications of the choice of the nonlinearity $R$ on results had often been raised in the engineering literature (see, e.g. [19]), and have occupied researchers since the inception of the ill-posed models treated here. The results of this paper provide a step towards a precise, rigorous understanding of this question. In particular, it is shown that for some nonlinearities coarsening can be faster than for (6). 


\section{Description of the system}

\subsection{Preliminaries}

We consider the unit-spaced lattice, $\mathbb{L}=\{1,2, \ldots, N\}^{d}$, where $d$ is the dimension. We identify this lattice with $\mathbb{Z}^{d}$ on which for any integers $1 \leqslant i_{j} \leqslant N$ and $k_{j}$ the point $\left(i_{1}+k_{1} N, \ldots, i_{d}+k_{d} N\right)$ is identified with $\left(i_{1}, \ldots, i_{d}\right)$. In other words, we use the periodic boundary conditions. We denote lattice configurations by $\mathcal{L}:=\{v: \mathbb{L} \rightarrow \mathbb{R}\}$. Of particular interest later on will be nonnegative configurations $\mathcal{P}:=\{v: \mathbb{L} \rightarrow[0, \infty)\}$.

We use the following notation:

$$
v \cdot w:=\sum_{q \in \mathbb{L}} v_{q} w_{q}
$$

We introduce the partial derivatives

$$
\left(\partial_{i}^{+} v\right)_{q}:=v_{q+e_{i}}-v_{q} \quad \text { and } \quad\left(\partial_{i}^{-} v\right)_{q}:=v_{q}-v_{q-e_{i}}
$$

and the corresponding gradients

$$
\nabla^{+} v=\left(\partial_{1}^{+} v, \ldots, \partial_{d}^{+} v\right) \quad \text { and } \quad \nabla^{-} v=\left(\partial_{1}^{-} v, \ldots, \partial_{d}^{-} v\right) .
$$

Note that the following summation by parts formula holds: for any integer $1 \leqslant i \leqslant d$

$$
\partial_{i}^{+} v \cdot w=-v \cdot \partial_{i}^{-} w
$$

The discrete Laplacian is

$$
\Delta v=\sum_{i=1}^{d} \partial_{i}^{-} \partial_{i}^{+} v
$$

By $|\mathbb{L}|=N^{d}$ we denote the number of nodes in the lattice. For any $\mathbb{M} \subset \mathbb{L}$ we define

$$
\bar{\sum}_{q \in \mathbb{M}} v_{q}:=\frac{1}{|\mathbb{L}|} \sum_{q \in \mathbb{M}} v_{q} .
$$

We denote the average value of configuration $v$ by

$$
\bar{v}:=\overline{\sum_{q \in \mathbb{L}}} v_{q} .
$$

Moreover, throughout the paper we use the symbols with bars to denote averages over the lattice $\mathbb{L}$.

We denote by $\mathcal{Z}$ the set of configurations with average zero: $\mathcal{Z}:=\{v \in \mathcal{L}: \quad \bar{v}=0\}$. On the set $\mathcal{Z}$ we introduce the discrete $H^{-1}$ norm in the following way: note that given $s \in \mathcal{Z}$ there exists unique, up to constant, solution $p$ of the discrete Laplace equation

$$
-\Delta p=s \text {. }
$$

We define the $H^{-1}$ inner product by

$$
\left\langle s_{1}, s_{2}\right\rangle:=\sum_{q \in \mathbb{L}}\left(\nabla^{+} p_{1}\right)_{q} \cdot\left(\nabla^{+} p_{2}\right)_{q} .
$$

Integration by parts gives $\left\langle s_{1}, s_{2}\right\rangle=s_{1} \cdot p_{2}=p_{1} \cdot s_{2}$.

For completeness we now prove a lemma that gives another, perhaps more familiar, form of the $H^{-1}$ norm.

Lemma 1. For $s \in \mathcal{Z}$

$$
\|s\|=\sup _{\xi \neq \text { const. }} \frac{s \cdot \xi}{\sqrt{\sum_{q \in \mathbb{L}}\left|\nabla^{+} \xi_{q}\right|^{2}}} .
$$


Proof. By the definition of the norm and the Cauchy-Schwarz inequality

$$
\|s\|=\sup _{w \in \mathcal{Z}, w \neq 0} \frac{\langle s, w\rangle}{\|w\|} .
$$

Given $w \in \mathcal{Z}$ let $\xi \in \mathcal{Z}$ be the solution of the equation $-\Delta \xi=w$. The mapping $w \mapsto \xi$ is a bijection from $\mathcal{Z}$ to $\mathcal{Z}$. By the definition of the inner product

$$
\frac{\langle s, w\rangle}{\|w\|}=\frac{s \cdot \xi}{\sqrt{\sum_{q \in \mathbb{L}}\left|\nabla^{+} \xi_{q}\right|^{2}}} .
$$

Taking the suprema, and noting that the right-hand side does not change if we add a constant to $\xi$ yields the desired equality.

\subsection{Gradient flow}

The evolution problem we study is

$$
\dot{v}=\Delta R(v)
$$

with $v(0) \in \mathcal{P}$. The nonlinearities considered are described as follows:

$$
R=f^{\prime}
$$

where

$$
f \in C^{1}([0, \infty),[0, \infty)), \quad f \text { is nondecreasing, and } f(0)=f^{\prime}(0)=0 .
$$

It is important to note that the condition $f^{\prime}(0)=0$ ensures that $v(t) \in \mathcal{P}$ for all $t \geqslant 0$; see for example lemma 2 in [6].

To a lattice configuration $v \in \mathcal{P}$ we associate the energy

$$
E(v):=\sum_{q \in \mathbb{L}} f\left(v_{q}\right)
$$

and the corresponding mean energy $\bar{E}(v):=E(v) /|\mathbb{L}|$.

We claim that evolution (8) is a gradient flow of the energy (9) with respect to $H^{-1}$ inner product (7). To verify the claim we compute for arbitrary $s \in \mathcal{Z}$

$$
\langle\dot{v}, s\rangle=-\langle-\Delta R(v), s\rangle=-R(v) \cdot s=-f^{\prime}(v) \cdot s=-\operatorname{grad} E(v) \cdot s .
$$

We further assume that $f(z)>\mu z^{\alpha}$ for some $\mu>0, \alpha \in[0,1)$ and all $z$ large enough. While this is all we need for the upper bound on coarsening, $f$ 's that we have in mind are convex near 0 , concave for $z$ large, and have one inflection point. For such $f$ we expect the coarsening considered on the infinite lattice $\mathbb{Z}^{d}$ to persist for all time for generic, large enough data. If $f$ has large local minima, or if the data are small, then the coarsening process may stop in finite time. In such cases the upper bound provided would still hold, but would, clearly, not be optimal.

\section{Upper bound on coarsening}

To a configuration $v$ we associate a 'length' scale:

$$
L:=\frac{1}{\sqrt{|\mathbb{L}|}}\|v-\bar{v}\|=\sup _{\xi \neq \text { const. }} \frac{\bar{\sum}_{q \in \mathbb{L}}\left(v_{q}-\bar{v}\right) \xi_{q}}{\sqrt{\sum_{q \in \mathbb{L}}\left|\nabla^{+} \xi_{q}\right|^{2}}} .
$$


While $\bar{v}$ is defined as a scalar, we also use it to denote the constant-valued lattice configuration. The reason that the word length is between quotation marks is that $L$ is not, in general, proportional to a true length scale of a system during the coarsening stage. In particular, if one considers a configuration with typical distance between nearby spikes $\ell$ then

$$
L \sim \begin{cases}\ell & \text { if } d=1,2, \\ \ell^{d / 2} & \text { if } d \geqslant 2 .\end{cases}
$$

The scaling above can be obtained by estimating $\|v-\bar{v}\|$. Recall that the test function $\xi$ for which the supremum is achieved is the solution of $-\Delta \xi=v-\bar{v}$. However, instead of solving the equation, a good enough test function $\xi$ can be obtained by using the fundamental solution of the Laplace equation near each spike, and by connecting these appropriately. This motivates the choice of test functions in (23) and (30).

The fact that there is a crossover in scaling between $L$ and $\ell$, as the dimension changes from 1 to 2 , is at the core of the crossover in the rate of coarsening (5). We should also note that the above holds when $d=2$ up to a logarithmic correction. The source of this is in the slow decay of the gradient of the fundamental solution to Laplace equation in dimension 2.

\subsection{Heuristic argument for the rate of coarsening}

We use the gradient-flow structure of the equation to give a heuristic argument for the rate of coarsening. From the gradient-flow structure (10) of equation (8) it follows that

$$
\frac{\mathrm{d} E}{\mathrm{~d} t}=-\|\dot{v}\|^{2}
$$

Let $h$ be the typical height of spikes in a configuration. Note that

$$
h \sim \ell^{d} \text {. }
$$

Furthermore note that the mean energy scales with $h$ as

$$
\bar{E} \sim h^{\alpha-1} \text {. }
$$

Consider the time interval, $\Delta t$, during which the typical height of spikes grows from $h$ to $2 h$.

The change in the mean energy scales as

$$
\triangle \bar{E} \sim h^{\alpha-1} \text {. }
$$

During time $\triangle t$, a significant proportion of spikes shrink until they disappear. This suggests

$$
\|\dot{v}\| \sim \frac{1}{\Delta t}\|v-\bar{v}\|
$$

Using (13) and (11) gives

$$
\Delta \bar{E} \sim \frac{1}{|\mathbb{L}|} \Delta t\|\dot{v}\|^{2} \sim \frac{1}{|\mathbb{L}|} \frac{1}{\Delta t}\|v-\bar{v}\|^{2}=\frac{1}{\Delta t} L^{2} .
$$

Combining (12), (14) and (15) gives

$$
h^{\alpha-1} \sim \frac{1}{\triangle t} \cdot \begin{cases}h^{2} & \text { if } d=1, \\ h & \text { if } d \geqslant 2 .\end{cases}
$$

Since $\Delta h \sim h$ it follows

$$
h^{\prime} \sim \frac{\Delta h}{\Delta t} \sim \begin{cases}h^{\alpha-2} & \text { if } d=1, \\ h^{\alpha-1} & \text { if } d \geqslant 2 .\end{cases}
$$


Solving the equations gives

$$
h \sim \begin{cases}t^{\frac{1}{3-\alpha}} & \text { if } d=1, \\ t^{\frac{1}{2-\alpha}} & \text { if } d \geqslant 2 .\end{cases}
$$

We note that in dimension 2 the above rate holds up to a logarithmic correction. Note also that combining (12), (14) and (15) gives

$$
1 \lesssim \begin{cases}\bar{E}^{\frac{1}{1-\alpha}} L & \text { if } d=1, \\ \bar{E}^{\frac{1}{1-\alpha}} L^{2} & \text { if } d \geqslant 2 .\end{cases}
$$

\subsection{Dissipation inequality}

The dissipation inequality follows from the simple fact that the distance of a moving point, $v(t)$, to a fixed point, $\bar{v}$, on a manifold cannot change faster than the speed of the point, $\|\dot{v}\|$. In that sense it follows from general considerations given in [13] and [17], but due to its simplicity we present a proof.

Lemma 2 (Dissipation inequality). Let $v$ be a solution of (8). Then

$$
(\dot{L})^{2} \leqslant-\dot{\bar{E}}
$$

Proof. We compute

Therefore

$$
2 L \dot{L}=\frac{\mathrm{d} L^{2}}{\mathrm{~d} t}=\frac{2}{|\mathbb{L}|}\langle\dot{v}, v-\bar{v}\rangle .
$$

$$
\dot{L}^{2}=\frac{1}{|\mathbb{L}|} \frac{\langle\dot{v}, v-\bar{v}\rangle^{2}}{\|v-\bar{v}\|^{2}} \leqslant \frac{1}{|\mathbb{L}|}\langle\dot{v}, \dot{v}\rangle=-\frac{1}{|\mathbb{L}|} \operatorname{grad} E \cdot \dot{v}=-\dot{\bar{E}}
$$

\subsection{Interpolation inequality}

We first introduce model potentials

$$
F_{\alpha}(z):= \begin{cases}0 & \text { if } 0 \leqslant z \leqslant \rho, \\ z^{\alpha} & \text { if } z>\rho,\end{cases}
$$

where $\rho>0$ and $\alpha \in[0,1)$. We now prove the inequalities suggested above for general potentials $f \geqslant \mu F_{\alpha}$ for some $\mu>0$.

Note that there are three length scales in the system: $\mu, \bar{v}$ and $\rho$. To investigate the scaling of inequalities in these quantities, we express how constants in the inequalities depend on them.

Lemma 3 (Interpolation inequality). Assume $f \geqslant \mu F_{\alpha}$ for some $\mu>0, \alpha \in[0,1)$. Assume that $\bar{v}>\rho$. Let $\bar{E}_{f}$ be the associated mean energy and assume

$$
\bar{E}_{f}<\frac{1}{72} \mu \frac{(\bar{v}-\rho)^{2-\alpha}}{\bar{v}^{2(1-\alpha)}} \text {. }
$$

If $d=1$ then

$$
\bar{E}_{f} L^{1-\alpha} \geqslant \frac{1}{24} \mu(\bar{v}-\rho)^{\frac{3(1-\alpha)}{2}+1} \bar{v}^{-\frac{3(1-\alpha)}{2}} .
$$

If $d \geqslant 2$ then

$$
\bar{E}_{f} L^{2(1-\alpha)} \geqslant \frac{1}{24} \mu d^{-(1-\alpha)}(\bar{v}-\rho)^{3-2 \alpha} \bar{v}^{-(1-\alpha)} .
$$


We consider systems with positive 'excess height', $\bar{v}-\rho$; otherwise the system could have zero mean energy. The scaling of the inequalities in $\mu$ is simple: if an interpolation inequality $\bar{E}_{F_{\alpha}} L^{\beta} \geqslant \theta>0$ holds and $f \geqslant \mu F_{\alpha}$ then $\bar{E}_{f} L^{\beta} \geqslant \mu \theta$. Also the condition $\bar{E}_{f}<C \mu$ implies $\bar{E}_{F_{\alpha}}<C$. Therefore it suffices to consider the case $f=F_{\alpha}$. In the remainder of the proof we write $\bar{E}$ for $\bar{E}_{F_{\alpha}}$.

We prove the inequalities in two steps. We first prove a weak form of (15). We show that typical spike height is greater than $\bar{E}^{-1 /(1-\alpha)}$. That is, we prove that at least $2 / 3$ of the excess height $\tilde{v}:=(v-\rho)_{+}$is in such spikes. The statement of the lemma and its proof are independent of the dimension.

Lemma 4. Let

$$
\begin{aligned}
& h:=\left(\frac{\bar{v}-\rho}{3}\right)^{\frac{1}{1-\alpha}} \bar{E}^{\frac{-1}{1-\alpha}} \quad \text { and } \\
& \mathbb{S}:=\left\{q \in \mathbb{L}: \tilde{v}_{q}>h\right\} .
\end{aligned}
$$

Then

$$
\bar{\sum}_{q \in \mathbb{L} \backslash \mathbb{S}} \tilde{v}_{q} \leqslant \frac{1}{3}(\bar{v}-\rho)
$$

and thus

$$
\bar{\sum}_{q \in \mathbb{S}} \tilde{v}_{q} \geqslant \frac{2}{3} \bar{\sum}_{q \in \mathbb{L}} \tilde{v}_{q}
$$

Proof. Assume the statement does not hold. We show that if too much mass is in 'low spikes' then the energy cannot be low enough. We estimate

$$
\begin{aligned}
& \frac{\bar{v}-\rho}{3}<\overline{\sum_{q \in \mathbb{L} \backslash \mathbb{S}}} \tilde{v}_{q} \\
& \leqslant \bar{\sum}_{\left\{q: \rho<v_{q} \leqslant h+\rho\right\}} \tilde{v}_{q}^{1-\alpha} v_{q}^{\alpha} \\
& \leqslant \max _{q \in \mathbb{L} \backslash \mathbb{S}} \tilde{v}_{q}^{1-\alpha} \overline{\sum_{\left\{q: \rho<v_{q}\right\}}} v_{q}^{\alpha} \\
& \leqslant h^{1-\alpha} \bar{E}=\frac{\bar{v}-\rho}{3}
\end{aligned}
$$

Contradiction.

\section{Proof of lemma 3.}

Case $d=1$. To estimate $\|v-\bar{v}\|$ we use a test function modelled after the fundamental solution of the Laplace equation. Let $k=\left\lfloor\frac{\bar{v}-\rho}{8 \bar{v}^{2}} h+1\right\rfloor$, where $h$ was defined in (21). The test function is

$$
\xi_{q}=\left(1-\frac{1}{k} \operatorname{dist}(q, \mathbb{S})\right)_{+} .
$$

Recall from (11)

$$
L^{2}=\frac{1}{|\mathbb{L}|}\|v-\bar{v}\|^{2} \geqslant \frac{\left(\bar{\sum}_{q \in \mathbb{L}}\left(v_{q}-\bar{v}\right) \xi_{q}\right)^{2}}{\bar{\sum}_{q \in \mathbb{L}}\left|\nabla^{+} \xi_{q}\right|^{2}} .
$$


The test function is chosen to be as flat as possible, but localized enough for the term (26) to dominate (27). The smallness condition (18) on $\bar{E}$ implies

$$
h \geqslant 24^{1 /(1-\alpha)}(\bar{v}-\rho)^{-1} \bar{v}^{2} .
$$

We first bound the numerator in (24) from below. To that end, we estimate

$$
\begin{aligned}
& \overline{\sum_{q \in \mathbb{L}}} v_{q} \xi_{q} \geqslant \overline{\sum_{q \in \mathbb{S}}} v_{q} \xi_{q}=\overline{\sum_{q \in \mathbb{S}}} v_{q} \quad(\text { using (23)) } \\
& \geqslant \overline{\sum_{q \in \mathbb{S}}} \tilde{v}_{q} \geqslant \frac{2}{3} \overline{\sum_{q \in \mathbb{L}}} \tilde{v}_{q} \text { (by lemma 4) } \\
& \geqslant \frac{2}{3}(\bar{v}-\rho) \text {. }
\end{aligned}
$$

Then, we estimate using $\xi_{q} \leqslant \sum_{p \in \mathbb{S}}\left(1-\frac{1}{k} \operatorname{dist}(q, p)\right)_{+}$,

$$
\begin{aligned}
\sum_{q \in \mathbb{L}} \bar{v} \xi_{q} & \leqslant \bar{v} \frac{|\mathbb{S}|}{|\mathbb{L}|} \sum_{i=-k}^{k}\left(1-\frac{|i|}{k}\right)=\bar{v} \frac{|\mathbb{S}|}{|\mathbb{L}|} k \\
& \leqslant \frac{\bar{v}^{2}}{h} k \quad\left(\text { since } h|\mathbb{S}| \leqslant \sum_{q \in \mathbb{S}} v \leqslant \bar{v}|\mathbb{L}|\right) \\
& \leqslant \frac{\bar{v}^{2}}{h}\left(\frac{(\bar{v}-\rho)}{8 \bar{v}^{2}} h+1\right)=\frac{(\bar{v}-\rho)}{8}+\frac{\bar{v}^{2}}{h} \\
& \leqslant \frac{(\bar{v}-\rho)}{8}+\frac{(\bar{v}-\rho)}{24^{\frac{1}{1-\alpha}}}(\text { by bound }(25) \text { on } h) \\
& \leqslant \frac{(\bar{v}-\rho)}{6} .
\end{aligned}
$$

Putting (26) and (27) together gives

$$
\sum_{q \in \mathbb{L}}\left(v_{q}-\bar{v}\right) \xi_{q} \geqslant \frac{(\bar{v}-\rho)}{2} .
$$

We bound the denominator in (24) from above. From the construction of $\xi$ follows that $\xi_{q}=\sup _{p \in \mathbb{S}}\left(1-\frac{1}{k} \operatorname{dist}(q, p)\right)$, and thus $\left|\nabla^{+} \xi_{q}\right|^{2} \leqslant \sum_{p \in \mathbb{S}}\left|\nabla^{+}\left(1-\frac{1}{k} \operatorname{dist}(q, p)\right)\right|^{2}$.

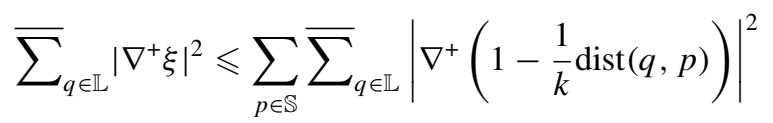

$$
\begin{aligned}
& \leqslant \frac{|\mathbb{S}|}{|\mathbb{L}|} 2 k \frac{1}{k^{2}} \leqslant 2 \frac{\bar{v}}{k h} \leqslant 16 \frac{\bar{v}^{3}}{(\bar{v}-\rho) h^{2}} .
\end{aligned}
$$

Therefore, putting (28) and (29) together implies

$$
L^{2} \geqslant \frac{1}{64} \frac{(\bar{v}-\rho)^{3} h^{2}}{\bar{v}^{3}} .
$$

Consequently, using the definition of $h$ in terms of $\bar{E}$,

$$
\bar{E}^{\frac{1}{1-\alpha}} L \geqslant\left(\frac{\bar{v}-\rho}{3}\right)^{\frac{1}{1-\alpha}} \frac{1}{h} \frac{1}{8} \frac{(\bar{v}-\rho)^{3 / 2} h}{\bar{v}^{3 / 2}} .
$$

This implies the claimed inequality. 


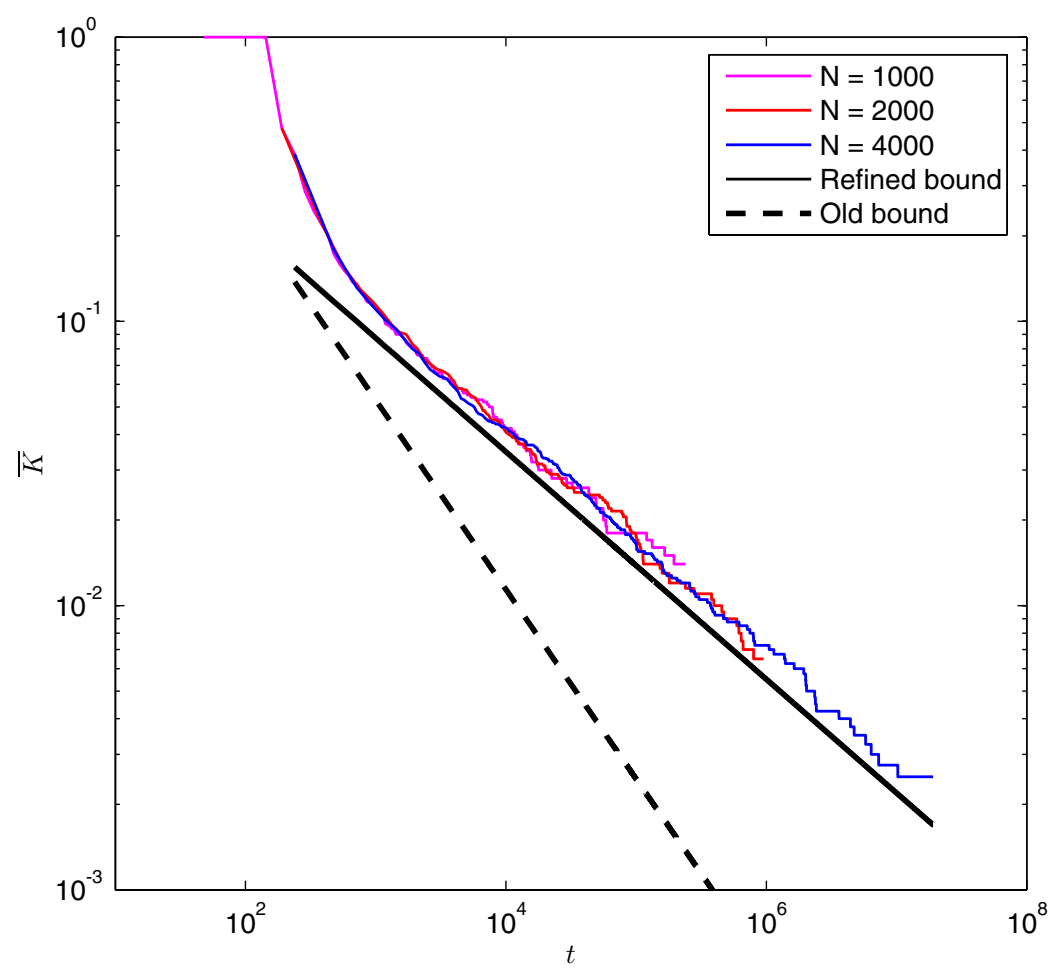

Figure 3. One dimensional experiments with the nonlinearity $f(\xi)=\left(1+|\xi|^{2}\right)^{\frac{1}{2}}$. Here, $\bar{K}$ denotes the spike density. The coarsening rate in terms of $\bar{K}$ indicated by the bound from [6], namely $\bar{K} \sim t^{-\frac{2}{3}}$, is represented by the dashed line, and is clearly far from optimal for this choice of $R$-not surprising since that result does not take into account the power law growth of the corresponding potential $f$ at infinity. The coarsening rate indicated by the new bound, namely $\bar{K} \sim t^{-\frac{2}{5}}$, is represented by the solid line and appears close to being optimal.

Case $d \geqslant 2$. This time, we take the characteristic function of $\mathbb{S}$ to be the test function:

$$
\xi:=\chi_{\mathbb{S}} .
$$

We note that a more careful choice, motivated by the fundamental solution of the Laplace equation, can be used if $d=2$ to obtain a logarithmic correction to the coarsening rate. As in the previous case, we estimate

$$
\begin{aligned}
& \bar{\sum}_{q \in \mathbb{L}}\left(v_{q}-\bar{v}\right) \xi_{q}=\overline{\sum_{q \in \mathbb{S}}}\left(v_{q}-\bar{v}\right) \geqslant \overline{\sum_{q \in \mathbb{S}}} \tilde{v}_{q}-\frac{|\mathbb{S}|}{|\mathbb{L}|} \bar{v} \\
& \geqslant \frac{2}{3} \overline{\sum_{q \in \mathbb{L}}} \tilde{v}_{q}-\frac{|\mathbb{S}|}{|\mathbb{L}|} \bar{v} \text { (by lemma 4) } \\
& \geqslant \frac{2}{3}(\bar{v}-\rho)-\frac{\bar{v}^{2}}{h} \geqslant \frac{\bar{v}-\rho}{2}(\text { by }(25)) \text {. }
\end{aligned}
$$

The construction (30) of $\xi$ implies for all $i=1, \ldots, d,\left|\partial_{i}^{+} \xi_{q}\right| \leqslant \sum_{p \in \mathbb{S}}\left|\partial_{i}^{+} \chi_{\{p\}}\right|$, and thus

$$
\bar{\sum}_{q \in \mathbb{L}}\left|\nabla^{+} \xi\right|^{2}=\sum_{i=1}^{d} \overline{\sum_{q \in \mathbb{L}}}\left|\partial_{i}^{+} \xi\right|^{2} \leqslant \frac{2 d|\mathbb{S}|}{|\mathbb{L}|} \leqslant 2 d \frac{\bar{v}}{h}
$$


Therefore,

$$
L^{2} \geqslant \frac{1}{8 d} \frac{(\bar{v}-\rho)^{2} h}{\bar{v}} .
$$

Consequently,

$$
\bar{E}^{\frac{1}{1-\alpha}} L^{2} \geqslant\left(\frac{\bar{v}-\rho}{3}\right)^{\frac{1}{1-\alpha}} \frac{1}{h} \frac{1}{8 d} \frac{(\bar{v}-\rho)^{2} h}{\bar{v}} .
$$

\subsection{Lower bound on energy}

We now establish the energy bound following the Kohn-Otto technique. ODE considerations by Kohn and Otto imply the following:

Proposition 1. Let $\bar{E}$ and $L$ be differentiable functions on $[0, \infty)$. Assume that for all $t \geqslant 0$

$$
\bar{E} \cdot L^{\beta} \geqslant \theta
$$

for some $\beta>0$ and $\theta>0$. Furthermore assume

$$
-\dot{\bar{E}} \geqslant \dot{L}^{2}
$$

for all $t$. Then for all $\sigma \in(1,1+2 / \beta)$

$$
\int_{0}^{T} \bar{E}^{\sigma}(t) \mathrm{d} t \gtrsim \int_{0}^{T}\left(t^{-\frac{\beta}{\beta+2}}\right)^{\sigma} \mathrm{d} t
$$

provided $T \gg L(0)^{\beta+2}$.

Remark 1. More precisely: let

$$
C(\beta, \sigma, \theta)=(\sigma-1)^{\frac{\sigma \beta}{\beta+2}}\left(\frac{\beta+2}{\beta+2-\sigma \beta}\right)^{1-\frac{\sigma \beta}{\beta+2}} \theta^{\frac{2 \sigma}{\beta+2}} .
$$

Given any $\sigma \in(1,1+2 / \beta)$, for all $\delta \in(0,1)$ there exists $T_{\delta}$ such that

$$
\int_{0}^{T} \bar{E}^{\sigma}(t) \mathrm{d} t \geqslant(1-\delta) C(\beta, \sigma, \theta) \int_{0}^{T}\left(t^{-\frac{\beta}{\beta+2}}\right)^{\sigma} \mathrm{d} t
$$

for all $T>T_{\delta} L(0)^{\beta+2}$.

The proof is analogous to the proof of proposition 1 in [17].

Theorem 1. Let $f$ be a potential such that $f \geqslant \mu F_{\alpha}$ for some $\mu>0$, where $F_{\alpha}$ is defined in (17). We consider initial configurations with $\bar{v}(0)>\rho$ and $\bar{E}(v(0))<e_{0}$, where $e_{0}$ is the right-hand side of (18). Let $v(t)$ be the solution of (8), and $\bar{E}(t):=\bar{E}(v(t))$ the associated mean energy (9).

If $d=1$ then

$$
\int_{0}^{T} \bar{E}^{\sigma}(t) \mathrm{d} t \geqslant \tilde{C}(\alpha, \sigma, \bar{v}(0), \rho, \mu) \int_{0}^{T}\left(t^{-\frac{1-\alpha}{3-\alpha}}\right)^{\sigma} \mathrm{d} t
$$

for $T \gg L(v(0))^{3-\alpha}$.

If $d \geqslant 2$ then

$$
\int_{0}^{T} \bar{E}^{\sigma}(t) \mathrm{d} t \geqslant \tilde{C}(\alpha, \sigma, \bar{v}(0), \rho, \mu) \int_{0}^{T}\left(t^{-\frac{1-\alpha}{2-\alpha}}\right)^{\sigma} \mathrm{d} t
$$

for $T \gg L(v(0))^{4-2 \alpha}$. 


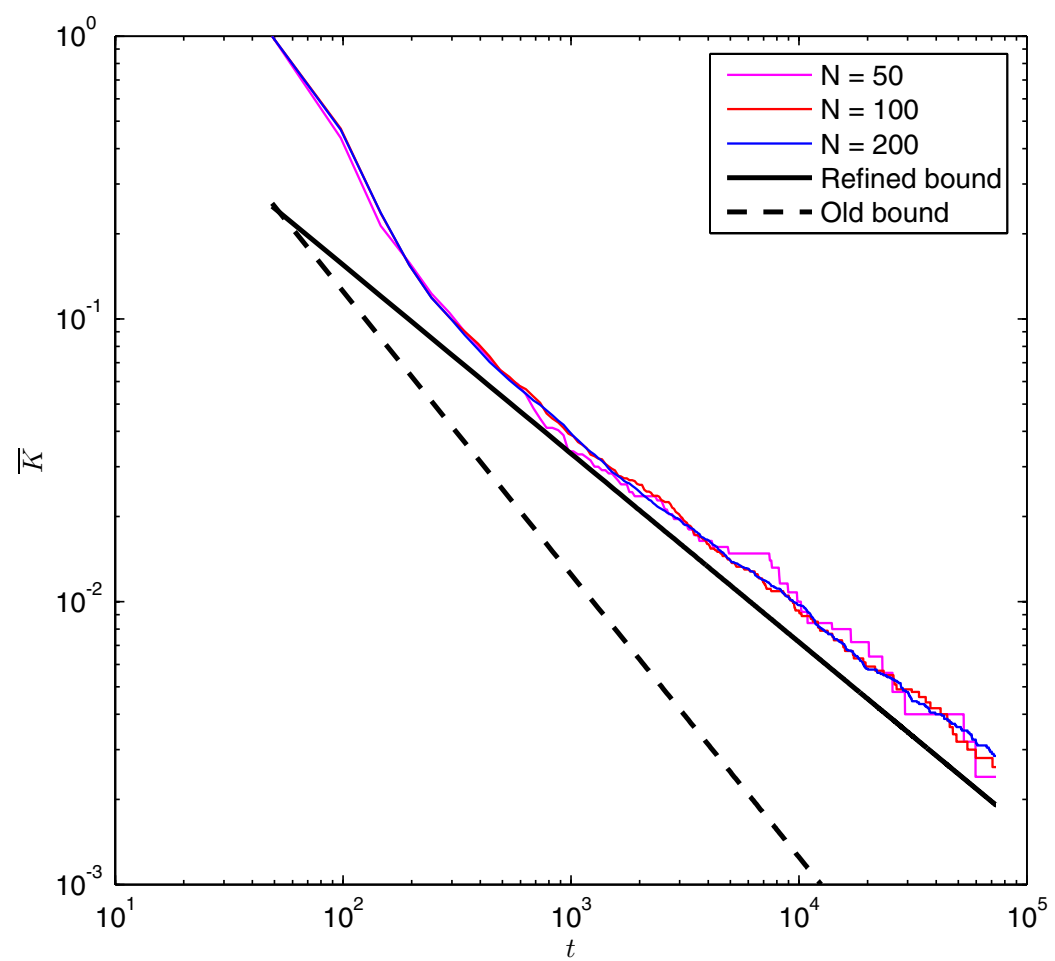

Figure 4. Experiments in two dimensions, with the nonlinearity $f(\xi)=\left(1+|\xi|^{2}\right)^{\frac{1}{4}}$. Once again the coarsening rate indicated by the new improved bound, namely $\bar{K} \sim t^{-\frac{2}{3}}$, represented by the solid line, seems much closer to being optimal than the bound from [6], which indicates the rate $\bar{K} \sim t^{-1}$, that does not take into account the power law growth of the corresponding potential $f$ at infinity.

The theorem follows from dissipation inequality (16) and the interpolation inequalities given in lemma 3 via the proposition 1 .

The precise constant $\tilde{C}$ in (34) and (35) is the one in (33), with $C$ from (32). The constant $\beta=1-\alpha$ if $d=1$ and $\beta=2(1-\alpha)$ if $d \geqslant 2$. The constant $\theta$ is the right-hand side of (19) if $d=1$ and of (20) if $d \geqslant 2$.

Remark 2. The inequalities above and the form of the energy suggest that for $f \sim \mu F_{\alpha}$ the typical spike height scales as

$$
h \sim \begin{cases}t^{\frac{1}{3-\alpha}} & \text { if } d=1, \\ t^{\frac{1}{2-\alpha}} & \text { if } d \geqslant 2 .\end{cases}
$$

So the coarsening is faster if $\alpha$ is closer to 1 .

Remark 3. The $\alpha=1$ case. Note that the gradient flows of energies corresponding to $f(z)$ and $f(z)-c z$, for any constant $c$, are identical. This is due to the fact that $\sum_{q \in \mathbb{L}} v_{q}$ is preserved by the evolution. Another way to see this is to note that adding a constant to $R$ does not change the evolution.

Thus when $f(z) \sim z$ for $z$ large, to study the coarsening one should renormalize the energy by subtracting an appropriate multiple of $z$. If $|f(z)-c z| \sim z^{\gamma}$ for all $z$ large, for some $\gamma \in[0,1)$ then the renormalized energy falls in the class of energies studied above. 


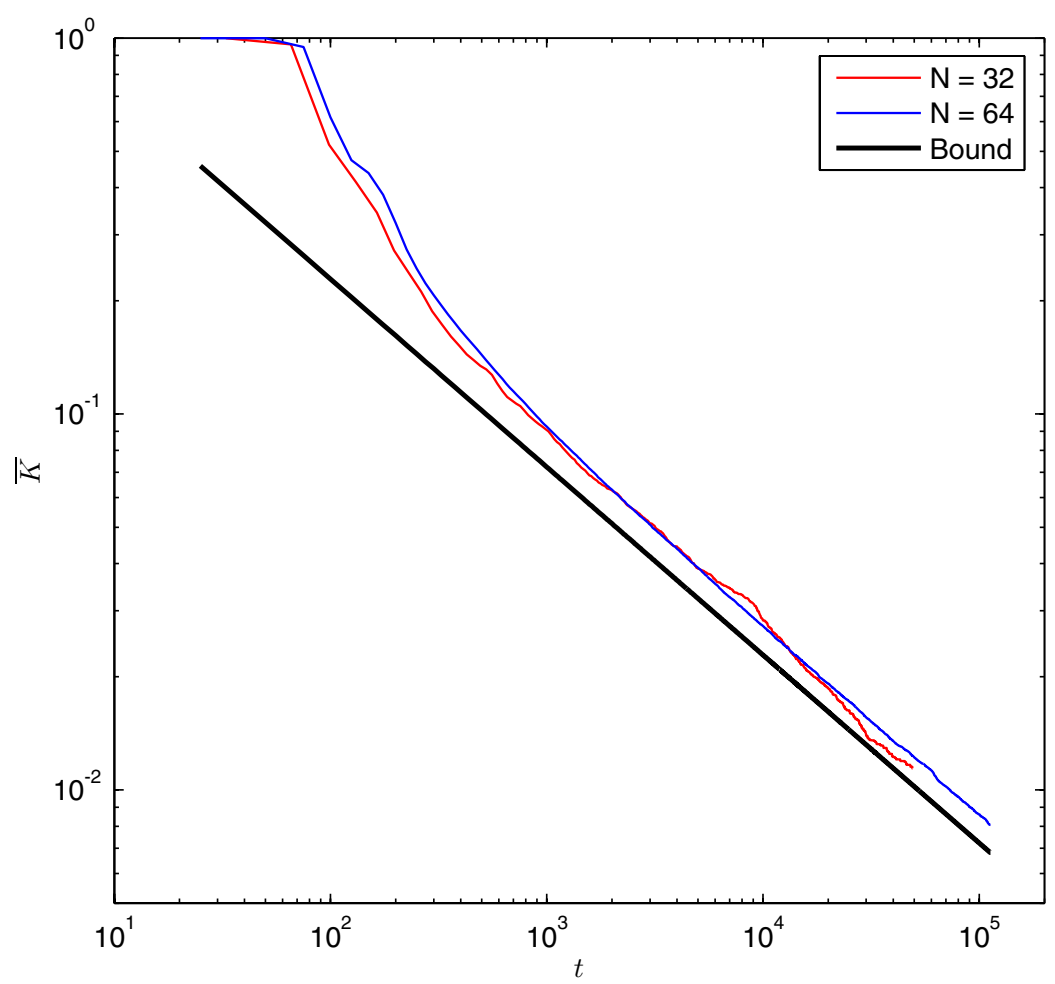

Figure 5. Experiments in three dimensions, with the nonlinearity $f(\xi)=\left(1+\xi^{2}\right)^{\frac{1}{4}}$. The coarsening rate indicated by theorem 1 , namely $\bar{K} \sim t^{-\frac{2}{3}}$, is represented by the solid line.

A particular consequence is that in the $\alpha=1$ case any rate of coarsening possible in the $\alpha<1$ range can be achieved. There can also be no coarsening at all, for example if $f(z)=z$.

Remark 4. It may be surprising then that the rate of coarsening that we predict increases as $\alpha \rightarrow 1^{-}$. The numerical experiments we describe in section 5 suggest that the rates in (36) are indeed optimal. Inspecting the constants in the inequalities can help explain this 'paradox'. For example, consider the constant $C$ in (32) for $\sigma=1+\frac{1}{\beta}$. That is for $1+\frac{1}{1-\alpha}$ if $d=1$ and for $\sigma=1+\frac{1}{2-2 \alpha}$ if $d \geqslant 2$. With this choice of $\sigma$, the first term in the expression for the constant $C$ in (32) becomes

$$
(\sigma-1)^{\frac{\sigma \beta}{\beta+2}}= \begin{cases}(1-\alpha)^{-\frac{2-\alpha}{3-\alpha}} & \text { if } d=1, \\ (2(1-\alpha))^{-\frac{3-2 \alpha}{4-2 \alpha}} & \text { if } d \geqslant 2 .\end{cases}
$$

In particular $C \rightarrow \infty$ as $\alpha \rightarrow 1^{-}$, which is consistent with potentially slower coarsening in the limiting case $\alpha=1$. See figures 7 and 8 for numerical experiments exploring this limit.

\section{Numerical results}

In this section we provide results of numerical experiments that corroborate the theory developed in section 4. Figures 3-5 show experiments at several resolutions in one, two 


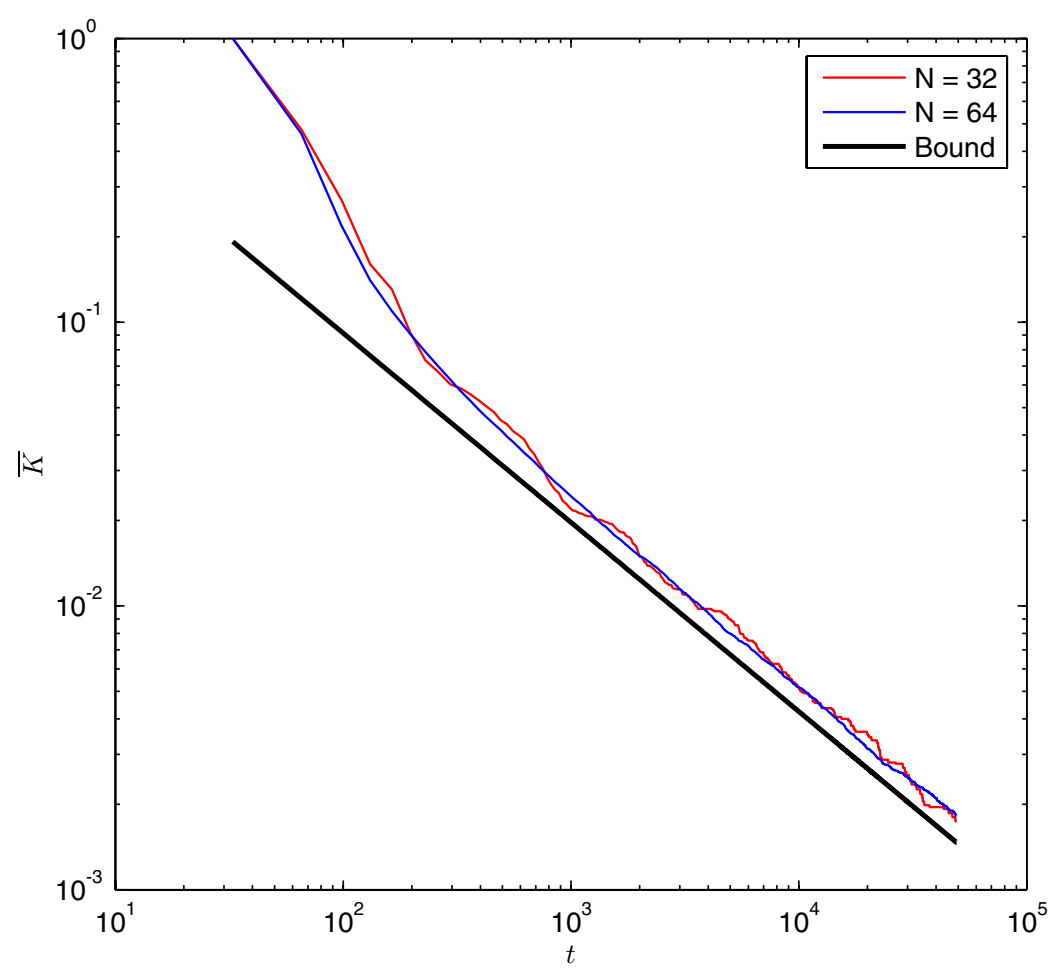

Figure 6. Experiments in three dimensions, with the nonlinearity $f(\xi)=\log \left(1+\xi^{2}\right)$. The coarsening rate predicted by theorem 1 , namely $\bar{K} \sim t^{-\frac{1}{2}}$, is indicated by the solid line.

and three space dimensions, respectively, with the evolution described by scheme (8) using the potential

$$
f(\xi)=\left(1+\xi^{2}\right)^{\frac{1}{4}}
$$

Theorem 1 applies to this flow with $\alpha=\frac{1}{2}$; the rigorous lower bound on time average of energy given by that theorem indicates the coarsening rates

$$
\bar{K} \sim \bar{E}^{\frac{1}{1-\alpha}} \sim \begin{cases}t^{-\frac{2}{5}} & \text { in 1D, } \\ t^{-\frac{2}{3}} & \text { in 2D and higher }\end{cases}
$$

where $\bar{K}$ is defined to be the density of spikes in the solution $v$ at any given time, i.e.

$$
\bar{K}:=\frac{\#\left\{q: v_{q} \geqslant \rho\right\}}{N^{d}}
$$

(recall that $N$ is the number of grid points in each coordinate direction). Here, $\rho$ is the inflection point of $f(\xi)$. The $\alpha$-independent lower bound on time average of energy given in [6] also applies to this flow, and indicates the rates

$$
\bar{K} \sim \bar{E}^{\frac{1}{1-\alpha}} \sim \begin{cases}t^{-\frac{2}{3}} & \text { in } 1 \mathrm{D}, \\ t^{-1} & \text { in } 2 \mathrm{D} .\end{cases}
$$

The dashed lines in figures 3 and 4 represent in terms of $\bar{K}$ the coarsening rate (39) implied by the rigorous bound from [6], whereas the solid line represents the bound (37) implied 


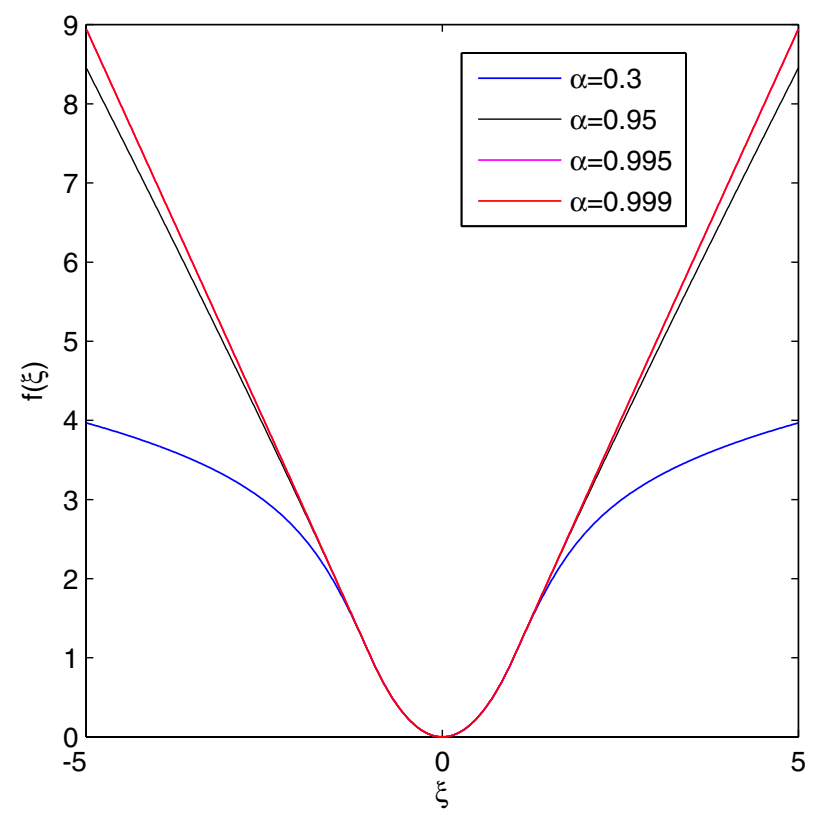

Figure 7. Energies used in the numerical investigation (see figure 8 for results) of the limit $\alpha \rightarrow 1^{-}$ discussed in remark 4 at the end of section 4 .

by theorem 1 of this paper. The new bound (37) appears close to being optimal in all of figures 3-5.

Figure 6 shows experiments in 3D with the standard Perona-Malik potential $f(\xi)=$ $\log \left(1+\xi^{2}\right)$. Theorem 1 gives the coarsening rate

$$
\bar{K} \sim \bar{E} \sim t^{-\frac{1}{2}}
$$

which agrees very well with the numerical results as indicated by the solid line: the bound appears very close to being optimal once again. In $1 \mathrm{D}$ and $2 \mathrm{D}$, with potential $f$, theorem 1 agrees with the rigorous bounds in [6], which were numerically demonstrated to be nearly optimal already in that paper.

Figures 7 and 8 show 1D experiments exploring the coarsening rate of scheme (8) in the limit $\alpha \rightarrow 1^{-}$in the potential

$$
f(\xi)= \begin{cases}\xi^{2} & \text { if }|\xi| \leqslant 1, \\ 1+2(|\xi|-1)\left(1+(|\xi|-1)^{2}\right)^{\frac{\alpha-1}{2}} & \text { if }|\xi|>1,\end{cases}
$$

which behaves as $|\xi|^{\alpha}$ for $|\xi|$ large, so that $f(\xi)$ approaches linear growth at $\infty$ as $\alpha \rightarrow 1^{-}$. This linear growth limit is in fact quite relevant for applications, as it essentially corresponds to the very popular total variation based image denoising model of Rudin, Osher and Fatemi [22]. As we approach this limit, any possible gains in energy through coarsening of large spikes (concentration of mass in few, tall spikes) becomes negligible. One would therefore expect the coarsening rate to vanish. However, as discussed in remark 4 of section 4 , the bound of theorem 1 indicates the coarsening rate

$$
\bar{K} \sim \bar{E}^{\frac{1}{1-\alpha}} \sim t^{-\frac{1}{3-\alpha}} \rightarrow t^{-\frac{1}{2}} \quad \text { as } \alpha \rightarrow 1^{-},
$$




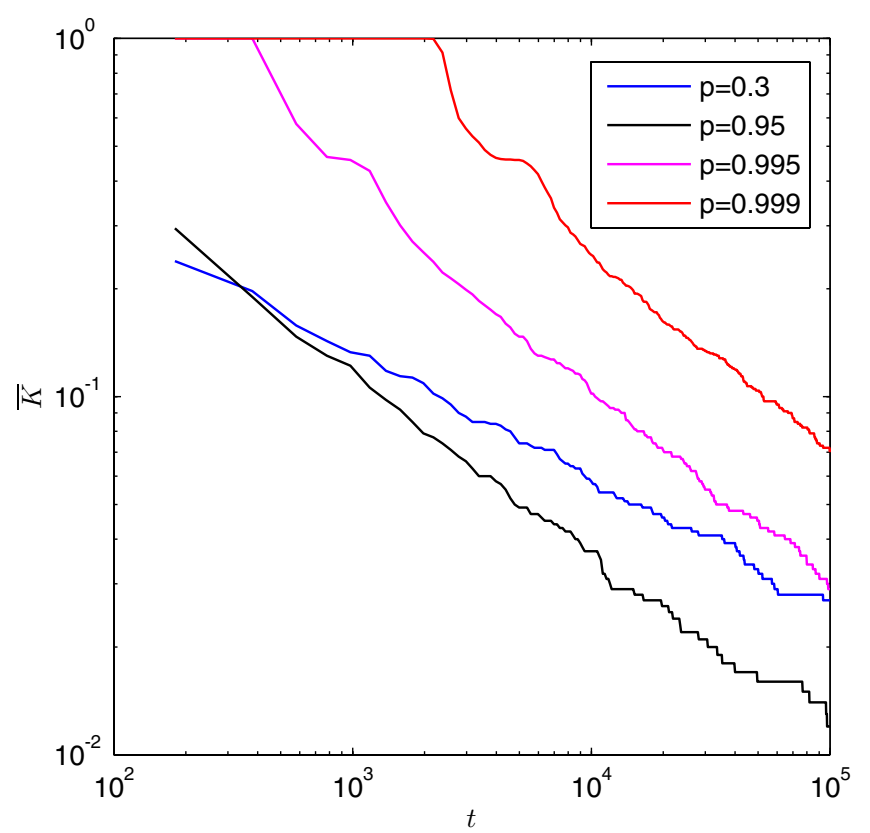

Figure 8. Numerical investigation of the limit $\alpha \rightarrow 1^{-}$discussed in remark 4 at the end of section 4 . Shown are the coarsening rates obtained in 1D by the family of energy densities plotted in figure 7 . As the growth at infinity of the energy densities approaches a linear rate (i.e. as $\alpha \rightarrow 1^{-}$), we see that the coarsening is delayed but its eventual rate is as predicted, namely $\bar{K} \sim t^{-\frac{1}{2}}$.

which suggests accelerated coarsening in this limit, contradicting initial expectations. The results shown in figure 8 help clarify this matter: coarsening rate indeed increases as $\alpha \rightarrow 1^{-}$, but it sets in at later and later times. As noted in the remark at the end of section 4 , this behaviour is hinted in the dependence on the parameter $\alpha$ of constants (32) appearing in the rigorous bound (33).

\section{Conclusion}

We presented rigorous upper bounds on the coarsening rate of spatially discrete versions of ill-posed diffusion equations that appear in a variety of applications. Our analysis was based on the approach of Kohn and Otto [13], and in a wide class of nonlinearities improved the bounds given in [6] for the same equations. Numerical results show that the upper bounds we established are in fact the rates observed in the experiments. Understanding the coarsening rate of these equations has direct implications in applications such as image processing.

\section{Acknowledgments}

Selim Esedoğlu was supported by NSF DMS-0748333, NSF DMS-0713767 and an Alfred P Sloan Foundation fellowship. Dejan Slepčev was supported by NSF DMS-0638481 and would also like to thank the Center for Nonlinear Analysis (NSF grants DMS-0405343 and DMS-0635983) for its support during the preparation of this paper. 


\section{References}

[1] Amann H 2007 Time-delayed Perona-Malik type problems Acta Math. Univ. Comenianae LXXVI 15-38

[2] Belahmidi A and Chambolle A 2005 Time-delay regularization of anisotropic diffusion and image processing M2AN 39 231-51

[3] Bellettini G and Fusco G 2003 A regularized Perona-Malik functional some aspects of the gradient dynamics EQUADIFF 2003, Proceedings of the International Conf. on Differential Equations ed H Broer et al (Hasselt, Belgium, July 2003)

[4] Bellettini G, Novaga M and Paolini E 2006 Global solutions to the gradient flow equation of a nonconvex functional SIAM J. Math. Anal. 37 1657-87

[5] Catte F, Lions P-L, Morel J-M and Coll T 1992 Image selective smoothing and edge detection by nonlinear diffusion SIAM J. Numer. Anal. 29 182-93

[6] Esedoğlu S and Greer J 2009 Upper bounds on the coarsening rate of discrete, ill-posed, nonlinear diffusion equations Comm. Pure Appl. Math. 62 57-81

[7] Esedoğlu S 2001 An analysis of the Perona-Malik scheme Comm. Pure Appl. Math. 54 1442-87

[8] Ghisi M and Gobbino M 2006 A class of local classical solutions for the one-dimensional Perona-Malik equation Preprint http://cvgmt.sns.it/papers/

[9] Ghisi M and Gobbino M 2007 Gradient estimates for the Perona-Malik equation Math. Ann. 337 557-90

[10] Gobbino M 2007 Entire solutions of the one-dimensional Perona-Malik equation Commun. Partial Diff. Eqns 32 719-43

[11] Horstmann D, Painter K J and Othmer H G 2004 Aggregation under local reinforcement from lattice to continuum European J. Appl. Math. 15 546-76

[12] Kichenassamy S 1997 The Perona-Malik paradox SIAM J. Appl. Math. 57 1328-42

[13] Kohn R V and Otto F 2002 Upper bounds on coarsening rates Commun. Math. Phys. 229 375-95

[14] Lizana M and Padron V 1999 A spatially discrete model for aggregating populations J. Math. Biol. 38 79-102

[15] Lou Y L, Tannenbaum A and Kaveh M 1996 Behavioral analysis of anisotropic diffusion in image processing IEEE. Trans. Image Process. 5 1539-53

[16] Nitzberg M and Shiota T 1992 Nonlinear image filtering with edge and corner enhancement IEEE Trans. Pattern Anal. Mach. Intell. 14 826-33

[17] Otto F, Rump T and Slepčev D 2006 Coarsening rates for a droplet model rigorous upper bounds SIAM J. Math. Anal. 38 503-29

[18] Painter K J, Horstmann D and Othmer H G 2003 Localization in lattice and continuum models of reinforced random walks Appl. Math. Lett. 16 375-81

[19] Perona P, Malik J and Shiota T 1995 Anisotropic diffusion Geometry-Driven Diffusion in Computer Vision ed B T Ter Haar Romeny (Dordrecht: Kluwer) pp 73-92

[20] Perona P and Malik J 1987 Scale space and edge detection using anisotropic diffusion Technical Report Department of EECS Technical Report, U.C. Berkeley

[21] Perona P and Malik J 1990 Scale-space and edge detection using anisotropic diffusion IEEE Trans. Pattern. Anal. Mach. Intell. 12 629-39

[22] Rudin L, Osher S J and Fatemi E 1992 Nonlinear total variation based noise removal algorithms Physica D 60 259-68

[23] Witelski T P, Schaeffer D G and Shearer M 2001 A discrete model for an ill-posed nonlinear parabolic PDE Physica 160 189-221

[24] Zhang K 2006 Existence of infinitely many solutions for the one-dimensional Perona-Malik model Calc. Var. Partial Diff. Eqns 26 171-99 